\title{
Intramitochondrial serine protease activity of Omi/ HtrA2 is required for caspase-independent cell death of human neutrophils
}

\author{
Cell Death and Differentiation (2004) 11, 937-939. doi:10.1038/sj.cdd.4401409 \\ Published online 26 March 2004
}

\section{Dear Editor,}

Omi/HtrA2 is a mitochondrial serine protease that has a proapoptotic role in mammalian cells. In the caspasedependent pathway of cell death, Omi/HtrA2 is released into the cytoplasm and binds to inhibitor of apoptosis proteins (IAPs), thus deactivating them and indirectly promoting the activation of caspases. Omi/HtrA2 can also initiate caspaseindependent cell death via its serine protease activity, but its substrates in this pathway are unknown. In the studies identifying the role of Omi/HtrA2 serine protease site, Omi/ $\mathrm{HtrA} 2$ relocated to the cytoplasm or was overexpressed cytoplasmically, leading to the postulation that Omi/HtrA2 enzymatic substrates are cytoplasmic. ${ }^{1-4}$

Studying the mechanisms of the TNF- $\alpha$-induced, caspaseindependent cell death in human neutrophils, ${ }^{5,6}$ we found that the serine protease activity of Omi/HtrA2 is required for, and its inhibition protects against, that type of neutrophil cell death. This was demonstrated in the following set of experiments with primary human neutrophils that were purified from the peripheral blood of healthy donors as described. ${ }^{5}$ Neutrophils have a short life-span and rapidly die during culturing in vitro without external stimulation. Already after a 6 -h incubation, about $15-20 \%$ of neutrophils has undergone spontaneous apoptosis, as was evident by Annexin-V-FITC binding (Figure 1a). Addition of TNF- $\alpha$ alone induced 'classical' caspase-dependent apoptosis (not shown; see Maianski et $a .^{5}$ ), whereas in the presence of the general caspase inhibitor zVAD this cytokine paradoxically enhanced the cell damage, inducing 'nonclassical' caspase-independent cell death in neutrophils with more than $50 \%$ of cells binding Annexin-V after $6 \mathrm{~h}$ (Figure $1 \mathrm{~b}$; also see Maianski et al. ${ }^{5}$ and Liu et al. $\left.{ }^{6}\right)$. Addition of ucf-101 $(20 \mu \mathrm{M})$, a specific inhibitor of Omi/HtrA2 serine protease activity, ${ }^{7}$ abrogated the death effects of the TNF- $\alpha+z V A D$ combination, significantly reducing the fraction of Annexin- $\mathrm{V}^{+}$neutrophils (Figure 1c). The extent of cell death measured by Annexin- $V$ was similar to that assessed by the mitochondrial changes using MitoTracker GreenFM (a fluorescent dye highly specific for mitochondria). In fresh neutrophils, virtually all cells displayed tubular mitochondria, which underwent clustering upon apoptosis, and the number of cells with the mitochondrial clusters corresponded to the number of apoptotic neutrophils. ${ }^{5}$ The mitochondrial morphology was estimated by a fluorescent microscope and indicated that $19 \pm 2 \%, 51 \pm 10 \%$ and
$20 \pm 6 \%$ (mean \pm S.E.M.; $n=3-4)$ of cells had clustered mitochondria in untreated, TNF- $\alpha+z V A D$ and TNF$\alpha+$ zVAD + ucf-101-treated cultures, respectively. Notably, ucf-101 prevented mitochondrial degradation induced by the TNF- $\alpha+z V A D$ treatment. These data indicate that Omi/HtrA2 also has a pro-death function in primary cells (neutrophils), as has previously been indicated upon overexpression in cell lines. ${ }^{1-4}$ However, ucf-101 did not protect neutrophils from spontaneous apoptosis or apoptosis induced by TNF- $\alpha$ alone, when tested up to $100 \mu \mathrm{M}$ at $18 \mathrm{~h}$ of culture, being at high concentrations even toxic to the cells (not shown). Thus, serine protease activity of Omi/HtrA2 is involved in the TNF- $\alpha$ induced, caspase-independent cell death pathway in neutrophils, but unlikely to play a role in 'classical', caspasedependent apoptosis. These findings emphasize a 'dual' nature of Omi/HtrA2 proapoptotic activity, which may rely on the IAP binding or on the serine protease properties. Apparently, for the usual apoptotic process in neutrophils (like the one in an 18-h culture) inactivation of IAPs is sufficient for Omi/HtrA2 to realize its proapoptotic potential, and the serine protease part is not essential. However, it has been shown that in 293 cells transfected with the active site mutant $\mathrm{Omi} / \mathrm{HtrA} 2$, which had no protease activity, caspase activation and cell death were markedly diminished, whereas transfection of the wild-type protein induced both events and resulted in significant reduction in the amount of XIAP in the transfected cells, which appeared to be a substrate of Omi/ HtrA2. ${ }^{8,9}$ Obviously, the way Omi/HtrA2 participates in cell death may differ in various cell types.

To further study the role of Omi/HtrA2 in the neutrophil cell death, we monitored the redistribution of this protein in subcellular fractions of neutrophils treated under various conditions. It has been shown in several cell lines that Omi/ $\mathrm{HtrA} 2$ is normally expressed in the mitochondria and relocates to the cytoplasm upon apoptosis. ${ }^{1-4}$ This was true for neutrophils as well, since Omi/HtrA2 was present in mitochondria in fresh neutrophils (Figure 1d, lane 6) and upon induction of apoptosis, for instance by incubation for $3 \mathrm{~h}$ with TNF- $\alpha$ and cycloheximide, when $70-80 \%$ of neutrophils became Annexin- $\mathrm{V}^{+}$, Omi/HtrA2 relocated to the cytoplasm (Figure 1d, lane 2) with a concomitant signal reduction in the mitochondrial fraction (Figure 1d, lane 7). The Omi/HtrA2 release into the cytoplasm was also observed in the overnight 


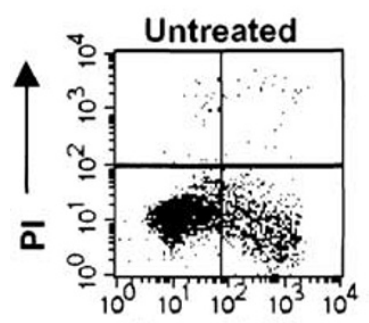

b

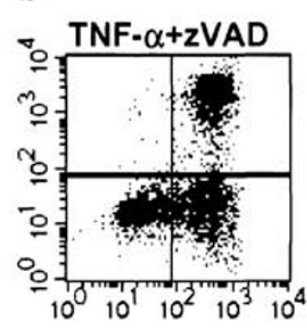

C

TNF- $\alpha+z V A D+u c f-101$

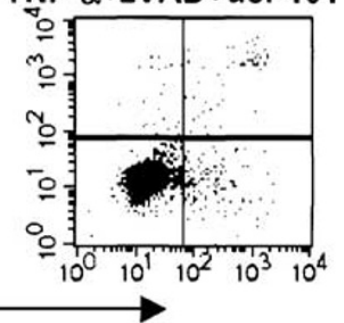

d Annexin V

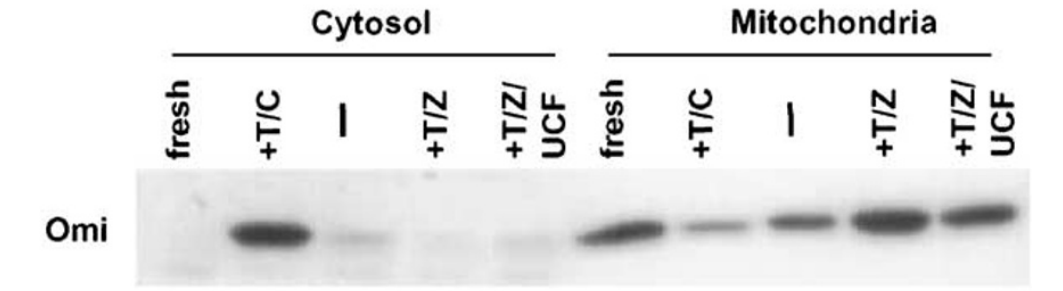

MnSOD

Apaf-1
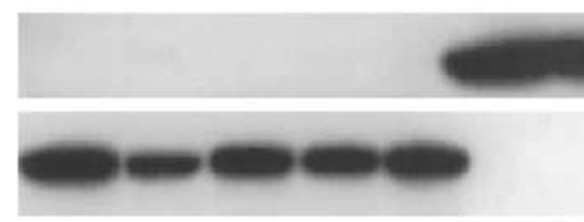

12

e
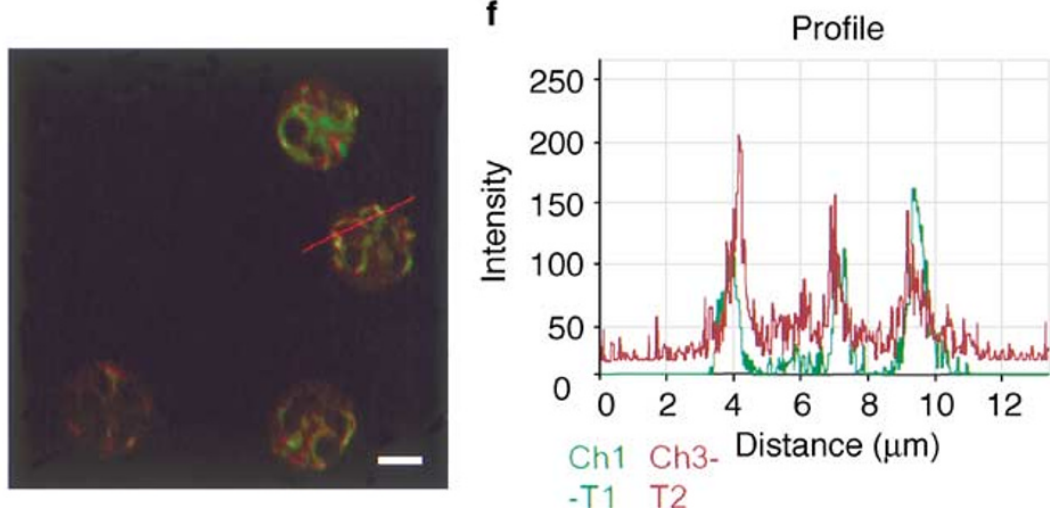

Figure 1 (a-c) Human neutrophils isolated from the peripheral blood of healthy donors were cultured for $6 \mathrm{~h}$ without additions (a), with a combination of $20 \mathrm{ng} / \mathrm{ml} \mathrm{TNF}-\alpha$ and $150 \mu \mathrm{M}$ zVAD-fmk (b) or with TNF- $\alpha / z V A D-f m k$ in combination with $20 \mu \mathrm{M}$ ucf-101 (c). Afterwards, the cells were stained with Annexin-V-FITC and PI and analyzed by FACScan as described. ${ }^{5}$ Representative plots are shown. The percentage of Annexin- $\mathrm{V}^{+}$cells was as follows (lower + upper right quadrants on the corresponding plot; mean \pm S.E.M.): A, $14 \pm 3 \%$; B, $48 \pm 15 \%$; C, $14 \pm 9 \%$. (d) Digitonin-based cell fractionation was performed on fresh neutrophils (fresh; lanes 1 and 6 ), in neutrophils incubated for $3 \mathrm{~h}$ with TNF- $\alpha(20 \mathrm{ng} / \mathrm{ml})$ and cycloheximide $(2 \mu \mathrm{g} / \mathrm{ml})$ to induce apoptosis (T/C; lanes 2 and 7$)$ and without additions for $6 \mathrm{~h}(-$, lanes 3 and 8$)$ or in cells cultured for $6 \mathrm{~h}$ with TNF- $\alpha+$ ZVAD-fmk (T/Z; lanes 4 and 9 ) or with TNF- $\alpha+z V A D-f m k$ and ucf-101 (T/Z + UCF; lanes 5 and 10). Thereafter, the cytosol (lanes 1-5) and the mitochondrial (lanes 6-10) fractions were subjected to SDS-PAGE. Western blot was performed with specific antibodies for the indicated proteins. Each neutrophil fraction represents $\sim 2 \times 10^{6}$ cells. Antibodies to MnSOD and Apaf-1 served as controls for purity of mitochondrial and cytosolic fractions, respectively. All manipulations were carried out as described..$^{10}$ (e) Fresh neutrophils were incubated with MitoTracker GreenFM $(100 \mathrm{nM})$ and ucf-101 $(20 \mu \mathrm{M})$ for 15 min at $37^{\circ} \mathrm{C}$, and intracellular localization of ucf-101 was determined by confocal laser scanning microscopy. Colocalization of MitoTracker GreenFM and ucf-101 gave a shift to yellow. Bar is $5 \mu \mathrm{m}$. (f) Colocalization of MitoTracker GreenFM (green line) and ucf-101 (red line) in the region on (e) marked by the red line is indicated semiquantitatively with the LSM510 image analysis software. (a-f) Each panel represents the results obtained from three to four experiments

cultured apoptotic neutrophils (not shown). After a 6-h incubation with TNF- $\alpha+z V A D$, Omi/HtrA2 remained in the mitochondria (Figure 1d, lanes 4 and 9) despite the induction of pronounced cell death (see Figure $1 \mathrm{~b}$ ). In the presence of the Omi/HtrA2 inhibitor ucf-101, when Annexin-V binding induced by TNF- $\alpha+$ zVAD stimulation was prevented, Omi/ HtrA2 also stayed in the mitochondria (Figure 1d, lanes 5 and
10). The purity of the subcellular fractions was confirmed by detection of Apaf-1 (cytoplasmic protein; Figure 1d, lanes 1-5) and MnSOD (the mitochondrial isoform of superoxide dismutase; Figure 1d, lanes 6-10).

The finding that $\mathrm{Omi} / \mathrm{HtrA} 2$ does not leave the mitochondria upon the TNF- $\alpha+z V A D$-induced cell death in neutrophils (Figure 1d, lanes 4 and 9) combined with the above-described 
ability of ucf-101 to completely block this death pathway (see Figure 1c) implies an important conclusion on 'nonclassical' cell death in primary cells. These data indicate that under certain conditions Omi/HtrA2 may mediate cell death through its serine protease properties from within the mitochondria. Indeed, when we studied the subcellular localization of ucf101 in neutrophils by utilizing the autofluorescent properties of this compound, ${ }^{7}$ it was found that the ucf-101 red autofluorescence and the signal from the mitochondrial marker MitoTracker GreenFM colocalized (Figure 1e; a shift in fluorescence to yellow depicts colocalization). Colocalization was confirmed by semiquantitative analysis with the LSM510 Image software (Figure 1f), which showed the peaks of ucf101 fluorescence (red) in close proximity with the peaks of MitoTracker fluorescence (green). This result indicates that ucf-101 localizes to the mitochondria and is in agreement with inhibitory activity being specific for Omi/HtrA2 and occurring within the mitochondria.

Another interesting issue that emerged from our data is that, in neutrophils, the mitochondrial permeabilization and release of $\mathrm{Omi} / \mathrm{HtrA} 2$ after the $\mathrm{TNF}-\alpha$ stimulation require caspase activity, because the presence of zVAD prevented those phenomena (Figure 1d, lanes 2, 7 and 4, 9, respectively), at least in a short-term culture. This is likely to be regulated by Bax redistribution from the cytosol (in intact cells) to the mitochondria (in apoptotic cells), which was prevented in TNF- $\alpha+z$ VAD-treated neutrophils but not in the neutrophils incubated with TNF- $\alpha$ alone. ${ }^{5}$ The targeting of mitochondria by Bax was also observed under the $3-\mathrm{h} \mathrm{TNF}-\alpha+$ cycloheximide treatment ${ }^{10}$ used in the present study. Hence, inhibition of caspases in the presence of TNF- $\alpha$ blocks the release of Omi/ HtrA2 from the mitochondria into the cytosol. However, the caspase inhibition under those condition does not prevent 'nonclassical' death signaling (see Figure 1b), which may still promote the Omi/HtrA2 function as a serine protease, but now inside the mitochondria.

Candidate intramitochondrial targets of the Omi/HtrA2 protease activity are illusive. The Omi/HtrA2-mediated proteolytic cleavage might inactivate and remove antiapoptotic molecules, as has been shown for IAPs, ${ }^{8,9}$ or it might be required for activation of precursor proteins whose function might be necessary for the caspase-independent neutrophil cell death. Since this type of cell death has been suggested to be mediated by the mitochondria-derived reactive oxygen species (ROS), ${ }^{5}$ it is logical to suppose that a mitochondrial antioxidant system, which normally inactivates an excess of
ROS, is destroyed in this experimental setting. Omi/HtrA2 might be involved in this inactivation, inhibiting for instance MnSOD, which is an antioxidant enzyme with high expression in neutrophils (Figure 1d, lanes 6-10; and see Maianski et $a .^{10}$ ) and important especially for prevention of the TNF- $\alpha$ induced damage.

In summary, we have shown that Omi/HtrA2 is expressed in mitochondria of fresh human neutrophils, but relocates into the cytosol upon apoptosis. More importantly, serine-protease activity of Omi/HtrA2 is required for the neutrophil caspase-independent, nonclassical cell death pathway induced by TNF- $\alpha$. Under these conditions, Omi/HtrA2 remains in mitochondria. This indicates that Omi/HtrA2 may support neutrophil cell death from within the mitochondria, likely by cleaving an intramitochondrial substrate(s). The physiological protease substrates for Omi/HtrA2 remain to be determined, although a mitochondrial protein that interacts with Omi's PDZ domain and is cleaved upon induction of mitochondrial stress is now being identified (A Zervos, unpublished data).

\section{E Blink $^{1,2}$, NA Maianski*1,2, ES Alnemri ${ }^{3}$, AS Zervos $^{4}$, $D$ Roos $^{1}$ and TW Kuijpers ${ }^{1,2}$}

1 Sanquin Research at CLB; and Landsteiner Laboratory, Academic Medical Center, University of Amsterdam, Amsterdam, The Netherlands;

2 Emma Children's Hospital, Academic Medical Center, University of Amsterdam, Amsterdam, The Netherlands;

${ }^{3}$ Center for Apoptosis Research, Kimmel Cancer Institute, Thomas Jefferson University, Philadelphia, PA, USA;

${ }^{4}$ Biomolecular Science Center (BSC), Department of Molecular Biology and Microbiology, University of Central Florida, Orlando, FL, USA

* Correspondence: NA Maianski, MD, Sanquin Research at CLB, and Landsteiner Laboratory, Academic Medical Center, University of Amsterdam, Plesmanlaan 125, 1066 CX Amsterdam, The Netherlands.

Tel: 31-20-512 3477; Fax: 31-20-512 3474;

E-mail: k.mayansky@sanquin.nl

1. Suzuki $Y$ et al. (2001) Mol. Cell 8: 613-621

2. Hegde R et al. (2002) J. Biol. Chem. 277: 432-438

3. Martins LM et al. (2002) J. Biol. Chem. 277: 439-444

4. Verhagen AM et al. (2002) J. Biol. Chem. 277: 445-454

5. Maianski NA, Roos D and Kuijpers TW (2003) Blood 101: 1987-1995

6. Liu CY et al. (2003) Blood 101: 295-304

7. Cilenti L et al. (2003) J. Biol. Chem. 278: 11489-11494

8. Srinivasula SM et al. (2003) J. Biol. Chem. 278: 31469-31472

9. Yang Q-H et al. (2003) Genes. Dev. 17: 1487-1496

10. Maianski NA et al. (2004) Cell Death Diff. 11: 143-153 